\title{
TWO RESULTS CONCERNING CARDINAL FUNCTIONS ON COMPACT SPACES
}

\author{
I. JUHÁSZ AND Z. SZENTMIKLÓSSY
}

\begin{abstract}
We show that for $X$ compact $T_{2}$ : (i) $d(X) \leqslant s(X) \cdot \hat{F}(X)$; (ii) if the pair $(\kappa, \hat{F}(X))$ is a caliber of $X$ then $\pi(X)<\kappa$.

These strengthen results of Sapirovskii from [3 and 5], respectively. Moreover, (i) settles a problem raised in [2] implying that there are no compact $T_{2} \kappa$-examples for any singular cardinal $\kappa$.
\end{abstract}

In this note we follow the notation and terminology of [1]. In particular, we let $\hat{F}(X)$ denote the smallest cardinal $\kappa$ such that $|S|<\kappa$ for any free sequence $S \subset X$.

THEOREM 1. If $X$ is compact $T_{2}$ then $d(X) \leqslant s(X) \cdot \hat{F}(X)$.

Proof. Let us put $s(X) \cdot \hat{F}(X)=\kappa$. Given any nonempty open set $U \subset X$ we can choose a family $\mathcal{C}(U)$ of open $F_{\sigma}$ sets in $X$ such that $U=\cup \mathcal{C}(U)$. But $X$ does not contain discrete subspaces of cardinality $\kappa^{+}$, hence, e.g. by 2.13 of [1], there is a subfamily $\mathscr{\Re}(U) \subset \mathcal{C}(U)$ and a subset $S(U) \subset U$ such that $|\Re(U)| \leqslant \kappa,|S(U)| \leqslant \kappa$ and $U \subset \cup \mathscr{B}(U) \cup \overline{S(U)}$.

Let us now assume, indirectly, that $d(X)>\kappa$. Then we also have $\pi(X)>\kappa$. Hence if $Q$ is a family of nonempty closed $G_{\delta}$ sets in $X$ with $|\mathscr{Q}| \leqslant \kappa$, then there is an open nonempty $U \subset X$ such that $A \backslash U \neq \varnothing$ for each $A \in \mathbb{Q}$. It follows easily from the compactness of $X$ that if $\mathscr{U}$ is a chain of open sets with this property, then $\cup \mathscr{U}$ possesses it as well. Thus by Zorn's lemma, we can fix an open set $W(\mathbb{Q})$ which is maximal with respect to the above property. Observe that then for every nonempty set $H$ open in $X \backslash W(\mathbb{Q})$, there is an $A \in \mathbb{Q}$ with $A \subset H \cup W(\mathbb{Q})$. Hence $\varnothing \neq$ $A \backslash W(\mathbb{Q}) \subset H$, i.e. $\{A \backslash W(\mathbb{Q}): A \in \mathbb{Q}\}$ is a $\pi$-network in $X \backslash W(\mathbb{Q})$. Consequently, we have

$$
d(X \backslash W(\mathbb{Q})) \leqslant|\mathbb{Q}| \leqslant \kappa .
$$

After these preparations we define by transfinite induction, families $\mathscr{G}_{\alpha}$ of closed $G_{\delta}$ subsets of $X$ for $\alpha \in \kappa$ with $\left|\mathscr{B}_{\alpha}\right| \leqslant \kappa$ as follows. If $\alpha \in \kappa$ and $\Re_{\beta}$ has been defined for all $\beta \in \alpha$, we consider the open set $W_{\alpha}=W\left(\cup\left\{\Re_{\beta}: \beta \in \alpha\right\}\right)$ and the family $\mathscr{B}\left(W_{\alpha}\right)$ of open $F_{\sigma}$ subsets of $W_{\alpha}$. For every $G \in \mathscr{B}\left(W_{\alpha}\right)$ we may then choose closed $G_{\delta}$ sets $F_{G}^{n}$ for $n \in \omega$ such that $G=\bigcup\left\{F_{G}^{n}: n \in \omega\right\} . \mathscr{B}_{\alpha}$ is then defined as the set of all nonempty finite intersections of members of the family

$$
\cup\left\{\mathscr{B}_{\beta}: \beta \in \alpha\right\} \cup\left\{X \backslash G: G \in \mathscr{B}\left(W_{\alpha}\right)\right\} \cup\left\{F_{G}^{n}: G \in \mathscr{B}\left(W_{\alpha}\right), n \in \omega\right\} .
$$


Clearly $\left|\mathscr{G}_{\alpha}\right| \leqslant \kappa$. This completes the induction.

Let us put

$$
Y=\cup\left\{\overline{S\left(W_{\alpha}\right)} \cup\left(X \backslash W_{\alpha}\right): \alpha \in \kappa\right\} ;
$$

since $\left|S\left(W_{\alpha}\right)\right| \leqslant \kappa$ and $d\left(X \backslash W_{\alpha}\right) \leqslant \kappa$, then $d(Y) \leqslant \kappa$ as well; hence, by our indirect assumption, $X \neq Y$.

We may thus pick a point $p \in X \backslash Y$. Then for every $\alpha \in \kappa$ there are $G_{\alpha} \in \Re_{B}\left(W_{\alpha}\right)$ and $n_{\alpha} \in \omega$ such that $p \in F_{\alpha}=F_{G_{\alpha}}^{n_{\alpha}} \subset G_{\alpha}$. Let us put, for $\alpha \in \kappa$,

$$
\mathscr{Z}_{\alpha}=\left\{F_{\beta}: \beta \leqslant \alpha\right\} \cup\left\{X \backslash G_{\beta}: \alpha<\beta<\kappa\right\} \text {. }
$$

We claim that $\mathscr{Z}_{\alpha}$ is centered. Indeed, if $\mathscr{Z} \in\left[\mathscr{Z}_{\alpha}\right]^{<\omega}$, then $\cap \mathscr{Z} \neq \varnothing$ follows by an easy induction on the number of the $X \backslash G_{\beta}$ 's in $\mathscr{Z}$ from our above construction.

Since the members of $\mathscr{Z}_{\alpha}$ are closed and nonempty, we can pick for each $\alpha \in \kappa$ a point $p_{\alpha} \in \cap \mathscr{Z}_{\alpha}$. But then $\left\{p_{\beta}: \beta \in \alpha\right\} \subset X \backslash G_{\alpha}$ and $\left\{p_{\beta}: \beta \in \kappa \backslash \alpha\right\} \subset F_{\alpha}$; hence

$$
\overline{\left\{p_{\beta}: \beta \in \alpha\right\}} \cap \overline{\left\{p_{\beta}: \beta \in \kappa \backslash \alpha\right\}}=\varnothing \text {. }
$$

This, however is a contradiction since $\left\{p_{\alpha}: \alpha \in \kappa\right\}$ is a free sequence in $X$ of size $\kappa \geqslant \hat{F}(X)$. Hence our proof is completed.

Theorem 1 is a strengthening of Šapirovskii's result saying that

$$
d(X) \leqslant s(X) \cdot t(X)^{+}
$$

for $X$ compact $T_{2}$, since, as is well known (see e.g. [1, 3.12]), for $X$ compact $T_{2}$ we have $F(X)=t(X)$. However the proofs of this given in $[3,4$ or 1$]$ do not seem to be modifiable to yield our result for the case in which $s(X) \cdot \hat{F}(X)=\hat{F}(X)=\kappa$ is a singular cardinal. That this case is of some independent interest is shown by the following result that solves a problem raised in [2] (and answered there only partially even for the case $\left.\operatorname{cf}(\kappa) \leqslant \omega_{1}\right)$.

COROllary. If $X$ is compact $T_{2}, \kappa$ is a singular cardinal, and $\pi(Y)<\kappa$ holds for each subspace $Y \subset X$ with $|Y| \leqslant \kappa$, then $\pi(X)<\kappa$ as well (or in the terminology of [2] there are no compact $T_{2} \kappa$-examples).

Proof. Clearly $X$ may have no discrete subspaces of cardinality $\kappa$. Hence we have $d(X) \leqslant s(X) \cdot \hat{F}(X) \leqslant \kappa$. But if $Y \subset X$ is dense with $|Y| \leqslant \kappa$, then by our assumption and 2.7 of $[1], \pi(X)=\pi(Y)<\kappa$.

To formulate our next result we recall that a pair $\langle\kappa, \lambda\rangle$ of cardinals is said to be a caliber of a space $X$ if for every family $\left\{G_{\xi}: \xi \in \kappa\right\}$ of nomempty open sets in $X$ there is a set $A \subset \kappa$ with $|A|=\lambda$ such that $\cap\left\{G_{\xi}: \xi \in A\right\} \neq \varnothing$.

THEOREM 2. If $X$ is compact $T_{2}$ and the pair $\langle\kappa, \hat{F}(X)\rangle$ is a caliber of $X$, then $\pi(X)<\kappa$.

Proof. Since the proof is quite similar to, but actually even simpler than, that of Theorem 1, we give only a sketch.

First, for any nonempty open set $U \subset X$ we fix a family $\bigodot(U)$ of open $F_{\sigma}$ 's in $X$ whose union is $U$. Second, assuming indirectly that $\pi(X) \geqslant \kappa$ and using that $\kappa>\omega$, for any family $Q$ of nonempty closed $G_{\delta}$ 's with $|Q|<\kappa$, we pick a nonempty open $F_{\sigma}$ 
set $W(\mathscr{Q})$ such that $A \backslash W(\mathbb{Q}) \neq \varnothing$ for all $A \in \mathbb{Q}$. Then, by transfinite induction, families $\mathscr{G}_{\alpha}$ of nonempty closed $G_{\delta}$ sets with $\left|\mathscr{B}_{\alpha}\right| \leqslant|\alpha|+\omega<\kappa$ are defined for $\alpha \in \kappa$ as follows. If $\alpha \in \kappa$ and $\mathscr{B}_{\beta}$ have been chosen for $\beta \in \alpha$, put

$$
W_{\alpha}=W\left(\cup\left\{\Re_{\beta}: \beta \in \alpha\right\}\right) \text {. }
$$

We can write

$$
W_{\alpha}=\bigcup\left\{F_{\alpha}^{n}: n \in \omega\right\},
$$

where the $F_{\alpha}^{n}$ are closed $G_{\delta}$ sets in $X$. Now, $\mathscr{B}_{\alpha}$ is defined as the set of all nonempty finite intersections of members of the family

$$
\cup\left\{\mathscr{B}_{\beta}: \beta \in \alpha\right\} \cup\left\{X \backslash W_{\alpha}\right\} \cup\left\{F_{\alpha}^{n}: n \in \omega\right\} .
$$

Clearly, $\left|\mathscr{G}_{\alpha}\right| \leqslant|\alpha|+\omega$.

Considering the family $\left\{W_{\alpha}: \alpha \in \kappa\right\}$ and using that, with $\lambda=\hat{F}(X)$, the pair $\langle\kappa, \lambda\rangle$ is a caliber of $X$, we can find a set $A \subset \kappa$ with $|A|=\lambda$ such that

$$
\cap\left\{W_{\alpha}: \alpha \in A\right\} \neq \varnothing \text {. }
$$

Let $p \in \cap\left\{W_{\alpha}: \alpha \in A\right\}$, and for each $\alpha \in A$ choose $n_{\alpha} \in \omega$ such that $p \in F_{\alpha}^{n_{\alpha}}=F_{\alpha}$ $\subset W_{\alpha}$. Exactly as in the proof of Theorem 1 we can see that for $\alpha \in A$ the family

$$
\mathscr{Z}_{\alpha}=\left\{F_{\beta}: \beta \in A \& \beta \leqslant \alpha\right\} \cup\left\{X \backslash W_{\beta}: \beta \in A \& \alpha<\beta\right\}
$$

is centered, and if $p_{\alpha} \in \cap \mathscr{Z}_{\alpha}$ for $\alpha \in A$, then $\left\{p_{\alpha}: \alpha \in A\right\}$ is a free sequence in $X$ of size $\lambda=\hat{F}(X)$, a contradiction. This completes the proof.

In [5] Sapirovskii proved that if $t(X)^{+}$is a caliber of a compact $T_{2}$ space $X$ then $\pi(X) \leqslant t(X)$. Since $\hat{F}(X) \leqslant F(X)^{+}=t(X)^{+}$and, moreover, if $F(X)^{+}$is a caliber of $X$, clearly so is the pair $\left\langle F(X)^{+}, \hat{F}(X)\right\rangle$ as well, this result is an immediate corollary of Theorem 2 .

\section{REFERENCES}

1. I. Juhász, Cardinal functions in topology-ten years later, Math. Centre Tracts, Vol. 123, Math. Centrum, Amsterdam, 1980.

2. I. Juhász and W. Weiss, Determination of $\pi$-weight by subspaces of singular cardinality, C. R. Math. Rep. Acad. Sci. Canada 3 (1981), 257-260.

3. B. Sapirovskii, Canonical sets and character. Density and weight in compact spaces, Soviet Math. Dokl. 15 (1974), 1282-1287.

4. Special types of embeddings in Tychonoff cubes. Subspaces of $\Sigma$-products and cardinal invariants, Topology, Vol. II (Proc. Fourth Colloq., Budapest, 1978), Colloq. Math. Soc. János Bolyai, Vol. 23, North-Holland, Amsterdam, 1980, pp. 1055-1086.

5. __. Cardinal invariants in compacta, Seminar on General Topology, Moscow, 1981, pp. 162-187. (Russian)

Mathematical Institute, Hungarian ACademy of SCIENCES, Reáltanoda 13-15, Budapest 1479 PF 128, HUNGARY 